\title{
Tumores fibroóseos de cavidades paranasales: Experiencia en el Hospital Clínico de la Universidad de Chile y revisión de la literatura
}

\author{
Fibro-osseous tumors of paranasal cavities: \\ Experience in the Hospital Clínico de la Universidad de Chile \\ and review of the literature \\ Cristóbal Chávez S $^{1}$, Karen García C ${ }^{1}$, Sergio Rojas N², Luis Barahona A¹, \\ Alfredo Naser G', Rodolfo Nazar S'. $^{1}$.
}

\begin{abstract}
RESUMEN
Los tumores de cavidades paranasales presentan una baja frecuencia. Dentro de éstos, entre los benignos destacan las lesiones fibroóseas que se caracterizan por el reemplazo de hueso normal por estroma celular fibroso. Dentro de estas lesiones se describen osteoma, displasia fibrosa y fibroma osificante. Se revisan 3 casos de pacientes del Hospital Clínico de la Universidad de Chile y se presenta una revisión bibliográfica en cuanto a las lesiones fibroóseas, su clínica, diagnóstico, imagenología y tratamiento.

Palabras clave: Lesión benigna fibroósea, osteoma, displasia fibrosa, fibroma osificante.
\end{abstract}

\begin{abstract}
The tumors of paranasal cavities present a low frequency. Among the benign tumors are fibro-osseous lesions characterized by the replacement of normal bone by fibrous cell stroma. Osteoma, fibrous dysplasia, and ossifying fibroma are described within these lesions. Three cases of patients from the Hospital Clínico de la Universidad de Chile are reviewed and a bibliographic review is presented regarding the fibro-osseous lesions, their clinical features, diagnosis, imaging and treatment.
\end{abstract}

Key words: Benign fibro-osseous lesion, osteoma, fibrous dysplasia of bone, ossifying fibroma.

\footnotetext{
1 Servicio de Otorrinolaringología, Hospital Clínico de la Universidad de Chile, Santiago, Chile.
}

2 Escuela de Medicina, Facultad de Medicina, Universidad de Chile, Santiago, Chile.

Los autores declaran no tener conflictos de interés.

Recibido el 22 de junio de 2019. Aceptado el 2 de diciembre de 2019. 


\section{INTRODUCCIÓN}

Los tumores de la cavidad nasal son de baja frecuencia, dando cuenta del 3\% de los tumores de la vía aéreo-digestiva superior y menos de $1 \%$ de todos los cánceres. Afecta con mayor frecuencia al sexo masculino, con una relación de 1,8:11. Dentro de los tumores malignos, el carcinoma escamoso es el más frecuente del tracto sinonasal, representando hasta el $48 \%$ de los casos, seguido por adenocarcinoma $(15 \%)$ y melanoma $(8 \%)^{2}$. Entre los tumores benignos destacan los papilomas sinonasales, angiofibroma nasofaríngeo juvenil y lesiones fibroóseas ${ }^{3}$. Estas últimas no han sido ampliamente descritas en cavidad nasal, por lo que resulta de interés su estudio y reporte.

Las lesiones fibroóseas son tumores caracterizados por el reemplazo de hueso normal por estroma celular fibroso, asociado a cantidades variables de focos de mineralización u osificación, que rara vez comprometen el tracto sinonasa 3.,. $^{3 .}$. Existen 3 tipos que, en orden según frecuencia, corresponden al osteoma, la displasia fibrosa y el fibroma osificante4. Debido a la baja de frecuencia de este tipo de lesiones, resulta relevante conocer el estudio diagnóstico y su manejo, por lo que presentamos una serie de 3 casos a continuación y con revisión de la literatura publicada.

\section{CASO CLÍNICO 1}

El primer caso corresponde a un hombre de 13 años, sin antecedentes mórbidos, con cuadro clínico de un año de epífora izquierda y diplopía ipsilateral. Al examen físico destacaba aumento de volumen en el canto medial del ojo izquierdo. Se realizó una tomografía computarizada (TC) de cavidades paranasales (CPN) que evidenciaba lesión expansiva esclerótica compatible con probable osteoma etmoidal con extensión orbitaria (Figura 1).

Se realizó cirugía endoscópica nasal (CENS) que incluyó antrostomía maxilar izquierda, etmoidectomía anteroposterior izquierda, y sinusotomía frontal con Draf II b izquierdo. La biopsia confirmó un osteoma (Figura 2). Se realizó una tomografía posoperatoria en la cual no se evidenció enfermedad residual (Figura 2). Sin recidiva a 1 año de seguimiento.

\section{CASO CLÍNICO 2}

La segunda paciente corresponde a una mujer de 27 años, sin antecedentes mórbidos, con historia de 2 años de pérdida de la agudeza visual izquierda, epífora, cefalea y rinorrea ipsilateral. Al examen físico se apreciaba exoftalmo izquierdo y

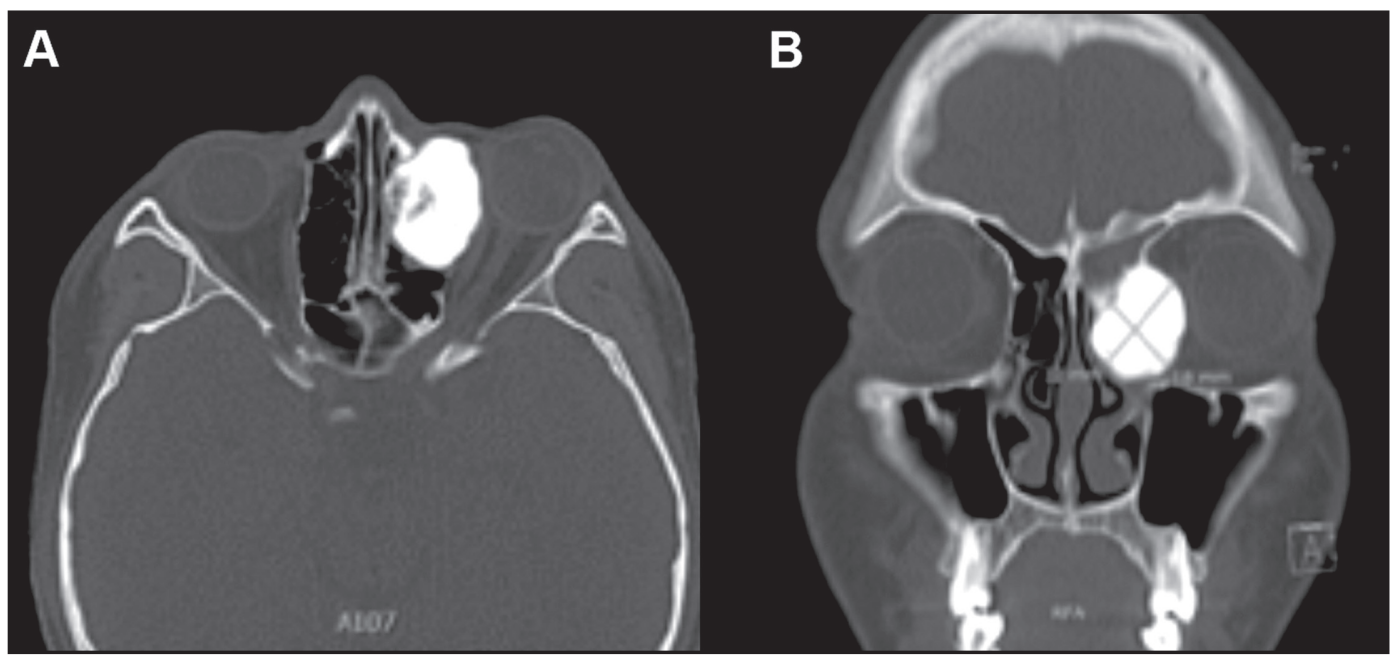

Figura 1. Tomografía computarizada de cavidades paranasales. (a) Corte axial y (b) corte coronal. En el laberinto etmoidal izquierdo, en relación con los dos tercios anteriores de la lámina papirácea se observa lesión expansiva esclerótica y ovalada, con características de osteoma. La lesión obstruye el conducto nasofrontal izquierdo condicionando la retención mucosa en el seno frontal izquierdo, además de generar severa estenosis del conducto lagrimal óseo ipsilateral, protruyendo hacia la órbita, desviando superolateralmente el músculo recto medial y el globo ocular, generando moderada proptosis. 


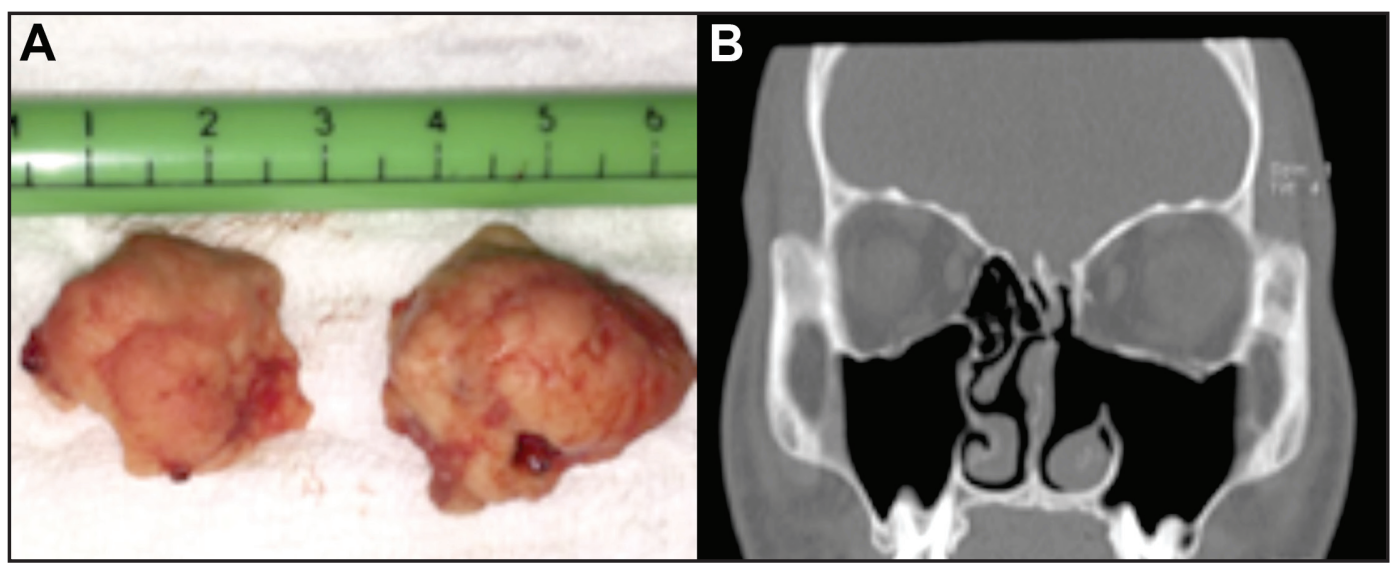

Figura 2. (a) Lesión informada como osteoma frontoetmoidal, el cual debió ser fragmentado para su retiro a través de la fosa nasal izquierda. (b) Tomografía computarizada posoperatoria sin lesión residual.

aumento de volumen en el epicanto y saco lagrimal ipsilateral. La motilidad ocular estaba conservada. Se realizó TC de CPN (Figura 3) con imagen de lesión expansiva nasoetmoidal izquierda.

Se realizó una CENS en fosa nasal izquierda guiada por navegación con imágenes de fusión tomografía computarizada con resonancia magnética (Figura 3) que incluyó maxilectomía medial, etmoidectomía anteroposterior con descompresión orbitaria (Figura 4) y del ápex orbitario, esfenoidotomía y sinusotomía frontal tipo Draf II b. La biopsia posoperatoria confirmaría el hallazgo de fibroma osificante juvenil de tipo psamomatoide. Sin recidiva en 6 meses de seguimiento.

\section{CASO CLÍNICO 3}

El tercer sujeto corresponde a un hombre de 15 años con cuadro de 6 meses de cefalea frontal izquierda intermitente, EVA 9/10, asociado a disestesia facial y descarga posterior. Al examen

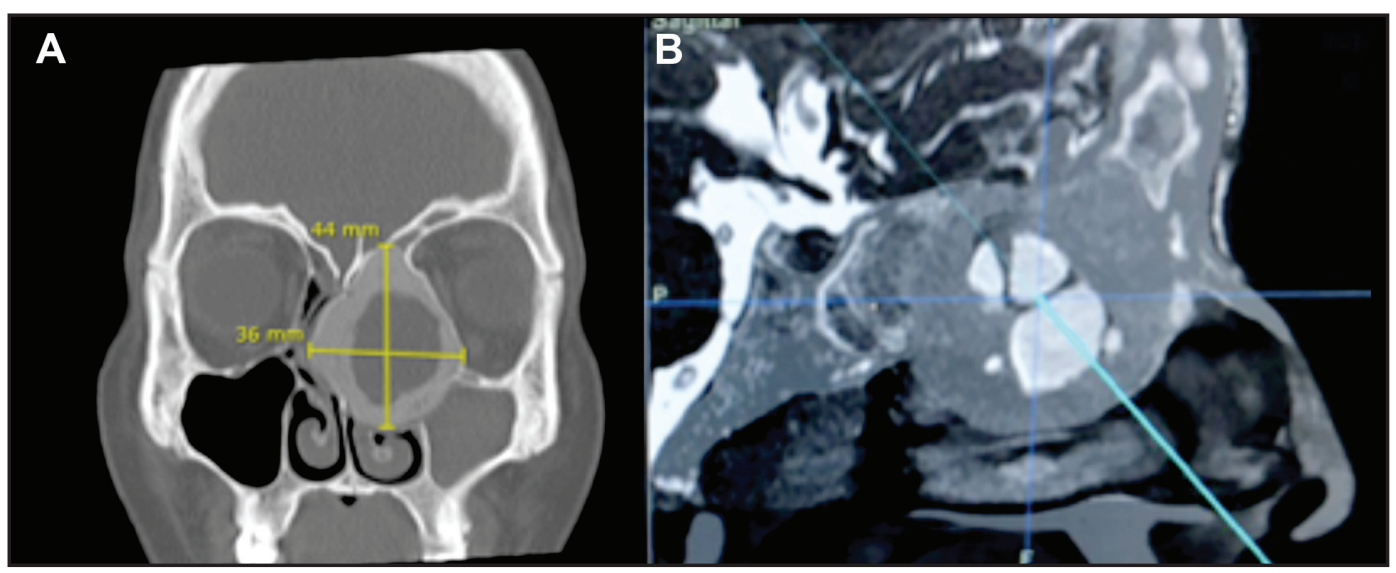

Figura 3. (a) Tomografía computarizada de cavidades paranasales, corte coronal. (b) Corte sagital de fusión TC-RM de cirugía asistida por video navegación. Lesión expansiva en la región nasoetmoidal izquierda que crece hacia la órbita desplazando latero-ventralmente el globo ocular, así como el músculo recto medial. Por su borde medial desplaza el tabique nasal hacia la derecha, ocupando parcialmente la región etmoidal. 


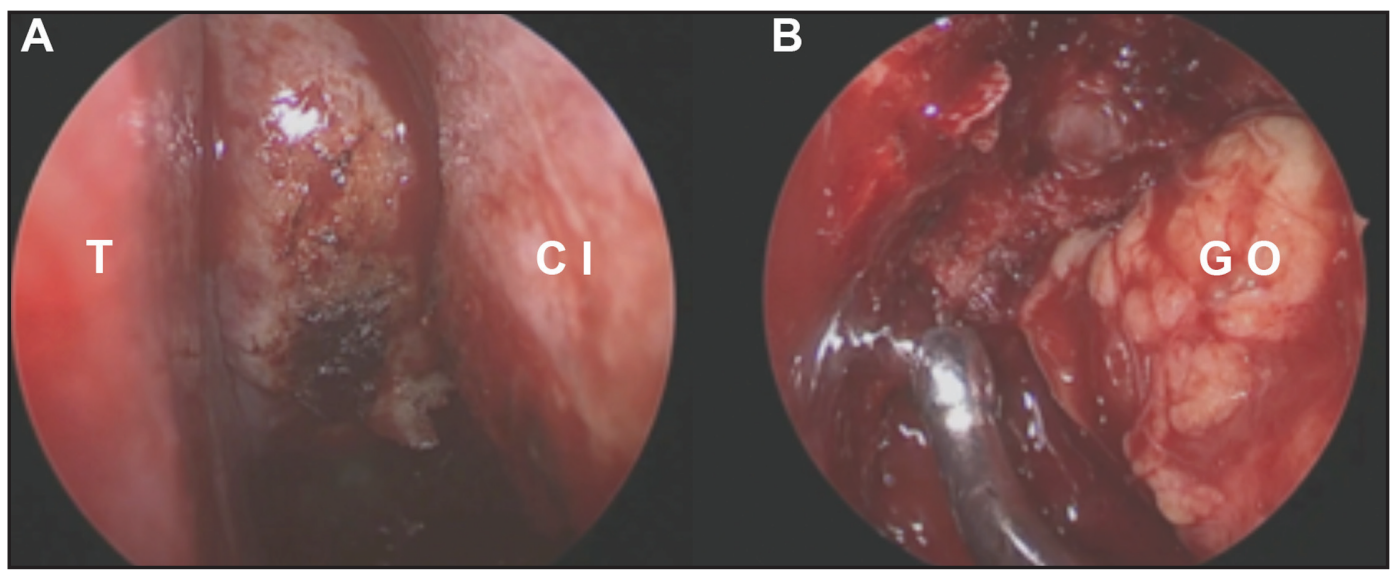

Figura 4. (a) Visión endoscópica de fibroma osificante preoperatorio. T =Tabique. $\mathrm{Cl}=$ Cornete inferior. (b) Visión endoscópica intraoperatoria en la cual se ha extraído lesión tumoral realizando descompresión orbitaria izquierda con exposición de la grasa (G0).

destacaba exoftalmo, descenso del globo ocular y abombamiento de la pared nasal lateral izquierda. El TC preoperatorio (Figura 5) evidenció una lesión ósea expansiva de seno frontal y etmoidal izquierdo con patrón de vidrio esmerilado. El estudio histológico posterior determinó que la lesión correspondía a displasia fibrosa monostótica. Se realizó una cirugía endoscópica con sinusotomía frontal Draf IIb más septectomía superior y fresado de la lesión guiado por navegación (Figura 5). En controles posoperatorios y seguimiento de 1 año el paciente no ha presentado recidiva de la lesión.

\section{DISCUSIÓN}

\section{Osteoma}

El osteoma es el tumor óseo más frecuente de la región facial, con una incidencia estimada desde 0,014\% hasta 3\%, según análisis tomográficos de cabeza y cavidades paranasales ${ }^{5}$. Es una lesión de crecimiento lento, que afecta con mayor frecuencia al sexo masculino, sin embargo, es más frecuente en mujeres en los casos reportados en cavidad nasal ${ }^{6}$. En caso de localizarse en el tracto

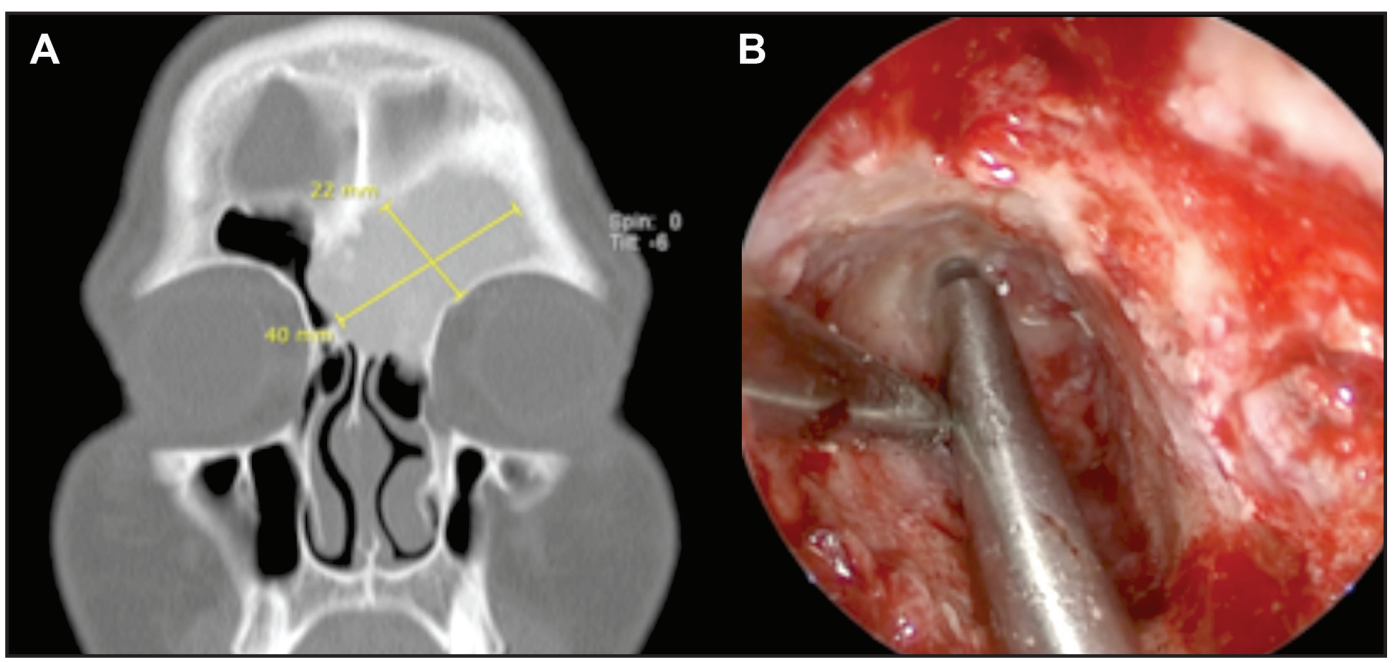

Figura 5. (a) Tomografía computarizada de cavidades paranasales preoperatoria que muestra lesión ósea expansiva con patrón en vidrio esmerilado. (b) Imagen endoscópica intraoperatoria en la cual se muestra el fresado de la lesión frontal. 
sinonasal, la ubicación más frecuente es el seno frontal, seguido por etmoidal, maxilar y esfenoidal, con escasos reportes en cavidad nasal. En cornetes nasales se han descrito 16 casos, de los cuales son 10 en cornete medio, 5 en inferior y 1 en superior $^{6}$. En relación a la etiología se han descrito 3 teorías: del desarrollo, traumática, e infecciosa. En la teoría del desarrollo se cree que células madre previamente silentes se activan y conducen a la formación descontrolada de hueso y, por otra parte, en las teorías traumática e infecciosa, se cree que el proceso inflamatorio sería el factor inductor de la formación de éstas ${ }^{3}$. Histológicamente se distinguen 3 tipos de osteoma: marfil, maduro y mixto. El osteoma marfil está compuesto de hueso cortical denso, el maduro de hueso esponjoso, mientras que el mixto tiene características de ambos $^{3}$.

Clínicamente una gran proporción son asintomáticos, pero la mayoría presenta síntomas como cefalea 0 dolor facial por obstrucción del drenaje de los senos paranasales. En osteomas más agresivos puede tener complicaciones orbitarias como en nuestro caso, siendo los síntomas principales epífora unilateral y diplopía, o complicaciones intracraneales, reportándose síntomas como diplopía, epífora, ceguera, fístula de líquido cefalorraquídeo, meningitis, neumatocele, absceso cerebral, entre otros ${ }^{3}$. En la TC se observan lesiones muy densas, homogéneas, bien delimitadas, con hueso adyacente normal, y se recomienda el uso de resonancia magnética (RM) ante sospecha de compromiso intracraneal ${ }^{5}$. Característicamente, si se instala un tubo de aspiración simultáneamente con un endoscopio nasal, se puede observar que, al tocar el tumor con la aspiración, el osteoma se mantiene rígido a la palpación? ${ }^{7}$.

En cuanto al tratamiento, la mayoría de las lesiones pequeñas y asintomáticas pueden ser manejadas de manera expectante, inicialmente con seguimiento cada 3 a 6 meses y luego anual en caso de mantenerse sin progresión en imágenes ${ }^{5}$. Sin embargo, ante un rápido crecimiento de las lesiones, aparición de sintomatología o deformidad facial se recomienda manejo quirúrgico, pudiendo ser por abordaje endonasal, externo o combinado. En nuestro paciente se prefirió el abordaje endoscópico nasal exclusivo debido a la baja morbilidad del procedimiento y la buena exposición de la lesión durante la cirugía. Con el desarrollo de la cirugía endoscópica se han reportado casos exitosos de cirugía asistida por imágenes con sistema de navegación principalmente en osteomas fronto-etmoidales, permitiendo mayor seguridad y precisión anatómica ${ }^{8}$. Los osteomas Iocalizados en la región frontal suelen tener un punto de inserción, mientras que los etmoidales no tienen un punto único de inserción y ésta pueda estar en toda la circunferencia del etmoides. Los casos descritos en cornetes nasales se han tratado el $50 \%$ mediante abordaje endonasal con resultados favorables, siendo el resto tratados alternativamente mediante incisión sublabial, gingival, resección craneofacial, técnica de Lynch o rinotomía lateral ${ }^{6}$. Existen dos reportes de casos en la literatura inglesa de osteomas en el septo nasal, ambos resueltos endoscópicamente ${ }^{7,9}$. El seguimiento en pacientes posoperados se realiza mediante endoscopía e imágenes de control en casos de que se sospeche clínicamente una recurrencia ${ }^{6}$. Es importante consultar por antecedentes de tumores o neoplasias colónicas familiares o personales, debido a su asociación con la poliposis adenomatosa familiar o síndrome de Gardner, enfermedad autosómica dominante caracterizada por poliposis intestinal difusa, fibromas, quistes sebáceos o epidermoides en piel, y osteomas en la región craneofacial ${ }^{10,11}$.

\section{Displasia fibrosa (DF)}

La DF representa 2,5\% de los tumores óseos y el $7,5 \%$ de las neoplasias óseas benignas ${ }^{12}$. Se debe a una mutación en el gen GNAS1, localizado en cromosoma 20q13.2-13.3, que codifica a un receptor acoplado a proteína Gs que provocaría una producción anormal de tejido fibroconectivo y aumento de expresión en células osteoprogenitoras indiferenciadas ${ }^{12}$. Histológicamente se caracteriza por un reemplazo de hueso normal por trabéculas óseas desordenadas y células fusiformes rodeadas de matriz fibrosa ${ }^{12}$. Se han descrito dos formas de DF: monostótica y poliostótica. La forma monostótica representa el 70\%-85\% de los casos, compromete solo un hueso y afecta el esqueleto facial en el $25 \%$ de los casos, siendo en la mayoría de los casos maxilar o mandíbula ${ }^{5}$. La forma poliostótica representa el $15 \%-30 \%$ de los casos, compromete más de 1 hueso y en el $3 \%$ 
se encuentra asociada al síndrome de McCuneAlbright y se caracteriza por la presencia de DF poliostótica asociada a pubertad precoz y manchas café con leche $e^{5,12}$. Se ha reportado que estas dos últimas formas comprometerían de manera frecuente el tracto sinonasal, afectando de manera decreciente al seno esfenoidal, frontal, maxilar, etmoidal y rara vez a cornete medio, inferior, piso nasal y septo nasal ${ }^{13}$

La DF se presenta en $75 \%$ de los casos antes de los 30 años, concordante con la edad de diagnóstico de nuestro paciente. Tradicionalmente se reporta que se estabilizaría al alcanzar la madurez esquelética, sin embargo, esto resulta controversial $^{5}$. No existen diferencias significativas respecto a 5 sexo ${ }^{3}$. La presentación clínica más habitual es el aumento de volumen, pudiendo asociarse a dolor, deformidad, fracturas, compromiso visual 0 auditivo ${ }^{12}$. En un estudio retrospectivo reciente de 28 casos de DF en el tracto sinonasal los síntomas más frecuentes fueron obstrucción nasal $(25 \%)$, alteración de la visión $(21 \%)$ y cefalea $(18 \%)$, reportándose la mayoría en seno esfenoidal $(50 \%)$, seguido por cavidad nasal $(39 \%)$ y seno etmoidal $(36 \%)^{12}$. Para el diagnóstico se requiere de una TC, que clásicamente muestra un patrón de vidrio esmerilado en la ventana ósea ${ }^{3}$, como fue en nuestro caso, el cual abombaba el seno frontal izquierdo. Sin embargo, se ha reportado que este patrón representaría sólo el 56\% de los casos, describiéndose también un patrón denso homogéneo $(23 \%)$ y variedad quística $(21 \%)$, dependiendo de la proporción de hueso mineralizado y tejido fibroso ${ }^{14}$. Se describe también el uso de citología por punción con aguja fina, que puede ser una herramienta preoperatoria útil en el diagnóstico diferencial de patologías más severas ${ }^{14}$.

En cuanto al tratamiento, no existe una recomendación general debido a la escasa documentación de casos en el tracto nasosinusal. En los casos asintomáticos, algunos autores recomiendan una evaluación periódica cada 6 meses ${ }^{12}$. En relación al tratamiento médico, se ha reportado el uso exitoso de bifosfonatos en cuanto a la reducción del dolor y remodelación ósea ${ }^{15}$, sin embargo poseen efectos adversos importantes que varían desde gastrointestinales (esofagitis y úlceras esofágicas), hipocalcemia, insuficiencia renal aguda, síntomas oculares como dolor, visión borrosa, uveítis y escleritis, siendo una de las más temidas la osteonecrosis de la mandíbula debiendo suspender el medicamento inmediatamente ${ }^{16-18}$. Se sugiere manejo quirúrgico en caso de lesiones sintomáticas, que crezcan durante el seguimiento 0 que causen un defecto estético, realizándose la mayoría de las veces resección endoscópica, ${ }^{5,14,15}$. En nuestro caso no se utilizó tratamiento médico y se optó por la vía endoscópica guiada por navegación debido a la alteración en los hitos anatómicos que producía la lesión.

Existen dos casos reportados en la literatura que comprometen el hueso nasal, recibiendo uno manejo expectante y otro quirúrgico mediante abordaje externo ${ }^{15}$. En un estudio reciente de DF en tracto sinonasal, casi el $90 \%$ de los pacientes fueron tratados con abordaje endonasal exclusivo de manera favorable, siendo solo necesario un abordaje combinado con incisión sublabial en tres casos de compromiso del seno maxilar ${ }^{12}$. Los autores de este trabajo recomiendan la remoción completa de lesión para evitar su recurrencia, por lo que se debe considerar abordajes alternativos para lograrlo como la rinotomía lateral, la incisión sublabial o resección craniofacial ${ }^{12}$.

Se ha descrito riesgo de malignización tanto de formas monostóticas como poliostóticas, estimándose en $0,5 \%$ en esta última forma y hasta $4 \%$ en síndrome de McCune Albright ${ }^{5,19}$. además, cabe destacar que resecciones parciales puede inducir crecimiento rápido de esta lesión o recurrencia, la cual puede llegar al $50 \%$ de los pacientes ${ }^{29}$, por lo que diversos autores sugieren que estos pacientes se mantengan en seguimiento periódico, poniendo especial atención al incremento rápido de la sintomatología dolorosa y un cambio repentino en la apariencia radiológica de la lesión, especialmente en cuanto a mineralización y destrucción de la cortical ósea se refiere ${ }^{19}$.

\section{Fibroma osificante (FO)}

Corresponde a la neoplasia fibroósea menos frecuente del tracto sinonasal ${ }^{4}$. En el $85 \%$ de los casos afecta a los huesos faciales, principalmente la mandíbula $(77 \%)^{20}$, sin embargo, el compromiso de otras localizaciones como la cavidad nasal o senos maxilar 0 etmoidal presenta característicamente un comportamiento más agresivo ${ }^{3}$. Su incidencia 
y etiología son desconocidas, pudiendo asignarse un rol al antecedente de trauma. Histológicamente se caracteriza por tener márgenes de tejido fibroso con cantidades variables de cuerpos psamommatoides mineralizados 0 calcificados, teniendo el FO convencional un patrón de mineralización heterogéneo, a diferencia de la $\mathrm{DF}^{3,4}$. Se diagnostica principalmente entre la segunda y cuarta décadas de vida, reportándose una frecuencia hasta cinco veces mayor en mujeres $^{3}$, situación concordante con nuestra paciente. Habitualmente el FO es asintomático, pudiendo diagnosticarse de manera incidental, pero en caso de presentarse síntomas se describen cefalea, obstrucción nasal, rinorrea, epistaxis, síntomas oculares y aumento de volumen facial, pudiendo llegar a presentar complicaciones como la formación de mucoceles, pérdida de agudeza visual e infecciones intracraneales ${ }^{21}$. La variante histológica juvenil es más agresiva, pudiendo extenderse hacia cavidades nasales, paranasales u órbita, con una edad de presentación más precoz ${ }^{3}$. Su transformación maligna es rara pero ha sido reportada, sin embargo, no se han descrito metástasis ${ }^{3}$. Algunos casos de la variedad juvenil se han asociado a tumores pardos de hiperparatiroidismo y a lesiones renales, siendo secundaria a una alteración en el gen supresor de tumores HRPT2, lo que resulta relevante puesto que aumenta el riesgo de malignización del F022,23.

En la TC, el FO se aprecia como una masa circular u ovalada de contenido heterogéneo rodeada de una cubierta ósea de crecimiento expansivo, pero no invasor ${ }^{20}$. En algunos casos se puede utilizar RM como en evaluación preoperatoria 0 ante sospecha de complicaciones, usualmente caracterizado por hipointensidad en T2 de áreas osificadas periféricas, con hiperintensidad en áreas centrales no osificadas ${ }^{24}$. En nuestra paciente se utilizó la RM tanto para evaluación preoperatoria, como para complemento intraoperatorio mediante navegación con fusión de imágenes de TC y RM (Figura 3). Si bien las imágenes radiológicas pueden llegar a ser altamente sugerentes de FO, idealmente se recomienda la confirmación con biopsia para descartar otras patologías como un sarcoma ${ }^{21}$. En caso de compromiso nasal, el FO puede apreciarse endoscópicamente como una masa redondeada de aspecto liso cubierto por mucosa ${ }^{21}$.
En cuanto al tratamiento, si las lesiones son asintomáticas y circunscritas a la mandíbula podrían únicamente ser controladas regularmente, sin embargo, si comprometen el tercio medio facial 0 el tracto nasosinusal habitualmente requieren resección quirúrgica dado su componente más agresivo ${ }^{3}$. El FO tiene una mayor tasa de recurrencia respecto a las otras lesiones fibroóseas, sucediendo entre el $30 \%$ a $58 \%$ de los casos en que se realiza remoción incompleta del tumor ${ }^{23}$, la cual es relativamente frecuente debido a la dificultad quirúrgica que otorga su alta vascularización y cantidad de adherencias durales y orbitarias ${ }^{4}$. De acuerdo a series de casos de FO de cavidades paranasales, habitualmente el tratamiento quirúrgico consiste en la remoción de la cubierta externa del tumor, fresando el hueso hasta alcanzar tejido sano de consistencia menos friable, lo que se puede realizar mediante vía abierta 0 , como se ha reportado últimamente, vía endonasal ${ }^{4}$. Sin embargo, de acuerdo a estas mismas series, este último abordaje tendría utilidad limitada en caso de extensión a nervio óptico, cara anterior de seno frontal y receso supraorbitario, o cara anterior de seno maxilar ${ }^{4}$. Los autores de este trabajo además no recomiendan el uso de técnicas de embolización del FO en cavidades paranasales a pesar de su alta vascularización, puesto que se relacionaría a complicaciones como la oclusión de la arteria central de la retina ${ }^{4}$. Se ha descrito el uso exitoso de tratamiento endoscópico en los escasos casos reportados de $\mathrm{FO}$ en cornete inferior, medio y concha bullosa ${ }^{20,25-28}$. Se recomienda realizar un seguimiento, consistente en endoscopías nasales frecuentes, además de TC sin contraste anualmente $^{18}$.

El diagnóstico diferencial de los tumores fibroóseos deber ser hecho con otras lesiones de crecimiento lento y sintomatología rinosinusal de predominio unilateral. Dentro de estas lesiones encontramos el tumor fibroso solitario, el cual debuta generalmente en la cuarta década de vida, de crecimiento lento, con una imagen tomográfica de partes blandas que puede contener calcificaciones en su interior. El diagnóstico definitivo se realiza con biopsia ${ }^{29}$. Las patologías producidas por hongos como la bola fúngica se caracterizan también por sintomatología unilateral asociado a velamiento con hiperdensidad prin- 
cipalmente en el seno maxilar ${ }^{30}$ y calcificaciones en su interior, siendo hipointensa en T1 y T2 en resonancia ${ }^{31}$.

Entre los diagnósticos diferenciales con patología maligna encontramos los carcinomas escamosos. En relación a ellos, el carcinoma cuniculatum es una entidad rara en senos paranasales, pero que se caracteriza por un patrón infiltrativo, sin embargo, las metástasis son infrecuentes por lo que se recomienda la escisión quirúrgica para su tratamiento ${ }^{32}$.

\section{BIBLIOGRAFÍA}

1. Turner JH, Reh DD. Incidence and survival in patients with sinonasal cancer: a historical analysis of population based data. Head Neck 2012; 34: 877-85.

2. Kuispens JHLP, Louwman MWJ, Peters R, Janssens GORJ, Burdorf AL, Coebergh J-WW. Trends in sinonasal cancer in The Netherlands: More squamous cell cancer, less adenocarcinoma: A population-based study 1973-2009. Eur J Cancer 2012; 48: 2369-74.

3. Lund VJ, Stammberger H, Nicolai P, Castelnuovo P, Beal T, Beham A, et al. European position paper on endoscopic management of tumours of the nose, paranasal sinuses and skull base. Rhinol Supp/2010; 22: 1-143.

4. Appiani MC, Verillaud B, Bresson D, Sauvaget E, Blancal J-P, Guichard J-P, et al. Ossifying fibromas of the paranasal sinuses: diagnosis and management. Acta Otorhinolaryngol Ital 2015; 35: 355.

5. Eller R, Sillers M. Common fibro-osseous lesions of the paranasal sinuses. Otolaryngol Clin North Am 2006; 39: 585-600.

6. VIRk RS, Sawhney S. Osteoma of the Middle Turbinate Presenting with Frontal Lobe Abscess and Seizure. J Clin Diagnostic Res 2017; 11: MD01.

7. KıM JS, NoH SJ, Ryu SH. Osteoma with actinomycosis in a nasal cavity: A case report. Medicine (Baltimore) 2017; 96: 51.

8. Fuentealba D, Naser A, Oliva C, Ritacco L, Miranda G, Nazar R. Manejo endoscópico de osteoma etmoidal con extensión orbitaria: A propósito de un caso. Rev Otorrinolaringol Cir Cabeza Cuello 2017; 77: 281-8.

\section{CONCLUSIÓN}

Las lesiones fibroóseas son un diagnóstico diferencial al momento de evaluar un paciente con síntomas otorrinolaringológicos de larga data. De éstos, el osteoma es el más frecuente. Para su enfrentamiento correcto resulta indispensable una imagen preoperatoria que revele el compromiso del tejido circundante a la hora de planificar el tratamiento, sea manejo conservador 0 una intervención quirúrgica, siendo la tomografía computarizada de CPN la herramienta idónea.

9. Yazici H, Soy FK, Kulduk E, Dündar R, Dogan $S$, KaYMAKÇI M, et AL. Endonasal endoscopic excision of a rare tumor: nasal bone osteoid osteoma. $J$ Craniofac Surg 2014; 25: e471-3.

10. Gardner EJ. Follow-up study of a family group exhibiting dominant inheritance for a syndrome including intestinal polyps, osteomas, fibromas and epidermal cysts. Am J Hum Genet 1962; 14: 376-90.

11. García EBG, Knoers NV. Gardner's syndrome (familial adenomatous polyposis): a cilia-related disorder. Lancet Oncol 2009; 10: 727-35.

12. Duan $C$, DaI Q, LIU Q, Yu H. Characteristics of sinonasal fibrous dysplasia: experience from a single department. Acta Otolaryngol 2018; 138: 50-5.

13. DeKlotz TR, Kim HJ, Kelly M, Coluins MT. Sinonasal disease in polyostotic fibrous dysplasia and McCune-Albright Syndrome. Laryngoscope 2013; 123: 823-8.

14. Park HJ, Cho M-S, Lee S-S. Fibrous dysplasia of the inferior turbinate. Int J Clin Exp Pathol 2013; 6: 531.

15. Wong G, Randhawa P, Stephens J, Saleh H. Fibrous dysplasia of the nasal bone: case reports and literature review. J Laryngol Otol 2013;127: 1152-4.

16. Jaimes, Miguel, Chaves Netto, Henrique Duque de Miranda, Olate, Sergio, Chaves, Maria das Graças Alfonso Miranda, \& Barbosa, José Ricardo de Albergaria. Bifosfonatos y Osteonecrosis de Ios Maxilares: Consideraciones Sobre su Tratamiento. International Journal of Morphology 2008; 26: 681-8. https://dx.doi.org/10.4067/ S0717-95022008000300028.

17. Fedele S, Porter SR, D'Aiuto F, et al. Nonexposed variant of bisphosphonate-associated osteo- 
necrosis of the jaw: a case series. Am J Med 2010; 123: 1060-4. doi:10.1016/j.amjmed.2010.04.033.

18. Khan AA, Morrison A, Hanley DA, et al. Diagnosis and management of osteonecrosis of the jaw: a systematic review and international consensus. J Bone Miner Res 2015; 30: 3-23. doi:10.1002/ jbmr.2405.

19. Qu N, Yao W, CuI X, Zhang H. Malignant transformation in monostotic fibrous dysplasia: clinical features, imaging features, outcomes in 10 patients, and review. Medicine (Baltimore) 2015; 94: e369. doi:10.1097/MD.0000000000000369.

20. Collin M, Roman S, Fernandez C, Triglia J-M, Nicollas R. Ossifying fibroma of the middle turbinate revealed by infection in a young child. Eur Ann Otorhinolaryngol Head Neck Dis 2014; 131: 193-5.

21. Manes RP, Ryan MW, Batra PS, Mendelsohn D, Fang YV, Marple BF. Ossifying fibroma of the nose and paranasal sinuses. Int Forum Allergy Rhinol 2013; 3: 161-8. doi:10.1002/alr.21067.

22. Yamashita Y, Akiyama T, Mizusawa N, Yoshimoto K, Gото M. A case of hyperparathyroidism-jaw tumour syndrome found in the treatment of an ossifying fibroma in the maxillary bone. Int J Oral Maxillofac Surg 2007; 36: 365-9.

23. Figueiredo LMG, de Oliveira TFL, Paraguassu GM, de Hollanda Valente R0, da Costa WrM, Sarmento VA. Psammomatoid juvenile ossifying fibroma: case study and a review. Oral Maxillofac Surg 2014; 18: 87-93.

24. Salina ACI, Souza PMM, Gadelha CMDC, Aguiar LB, Castro JDV, Barreto ARF. Ossifying fibroma: an uncommon differential diagnosis for T2-hypointense sinonasal masses. Radiol
Case Rep 2017; 12: 313-7. doi:10.1016/j. radcr.2017.03.019.

25. Caylakli $F$, Buyuklu F, Cakmak 0, Ozdemir $\mathrm{H}$, Ozluoglu L. Ossifying fibroma of the middle turbinate: a case report. Am J Otolaryngo/2004; 25: 377-8.

26. Galvan 0, Gassner EM, Neher A, Gunkel AR. Fibroosseous lesion of the middle turbinate: ossifying fibroma or fibrous dysplasia? J Laryngol Otol 2007; 121: 1201-3.

27. Cukurova I, Demirhan E, Karaman Y, Yigitbasi OG. Extraordinary pathologic entities within the concha bullosa. Saudi Med J 2009; 30: 937-41.

28. Pata YS, Ekici ID, Cihangiroglu M, Dogan M, Kocak I. Ossifying fibroma of the inferior turbinate. Kulak Burun Bogaz Ihtis Derg 2011; 21: 163-6.

29. García de Marcos Ja, Castillo Pardo de Vera JL, Arroyo Rodríguez S, Galdeano Arenas M, Calderón Polanco J, García de Marcos MJ et AL. Tumor fibroso solitario intraoral: análisis clinicopatológico e inmunohistoquímico. Rev Esp Cirug Oral y Maxilofac 2008; 30: 180-4.

30. Palma S, Heider C, Muñoz T, lagos A, Fonseca X, Callejas $C$, Gonzalez C. Bola fúngica de cavidades paranasales. Serie de casos. Rev Otorrinolaringol Cir Cabeza Cuello 2018; 78: 141-6.

31. Aribandi M, McCoy VA, Bazan III C. Imaging features of invasive and noninvasive fungal sinusitis: a review. Radiographics 2007; 27; 1283-96.

32. Sepúlveda I, Spencer L, Platin E, Bravo F, Sepulveda I, SPencer L, et AL. Maxillary Cuniculatum Carcinoma: a Case Report and. Int J Odontostomat 2012; 6: 281-4.

\footnotetext{
Correspondencia: Cristóbal Chávez S.

Hospital Clínico de la Universidad de Chile

Santos Dumont \#999, Independencia. Santiago, Chile

E mail: cristobal.chs@gmail.com
} 